\title{
Anestesia espinal parte III. Mecanismos de acción
}

\section{Spinal anesthesia part III. Mechanisms of action}

\author{
Héctor Lacassie Q. ${ }^{1}$, Fernando Altermatt C. ${ }^{1}$, María Jesús Irarrázaval M. ${ }^{2}$, Catalina Kychenthal L. ${ }^{2}$ \\ Juan Carlos De La Cuadra F. ${ }^{1}$
}

\begin{abstract}
Spinal anesthesia is a widely used technique in medical practice nowadays. Generally, nervous blockage is determined by three main factors. The first of them is the distribution of the local anesthetic in the cerebrospinal fluid (CSF), which can be affected by numerous factors, the most important of them being CSF volume. The second is absorption, which is greatest at the sites with higher drug concentration: this is the result of the accessibility, lipidic content and vascular irrigation of each area. The last of these factors is elimination, mediated mainly by the irrigation of the different compartments, and whose order differs from the mirror image of the onset's action order. The previously mentioned elements are the main sources of variation for the time needed to achieve desired effects, order in which fibers are affected and differential blockage. This text describes the principal mechanisms through which spinal anesthesia works, and the factors which can result in variations of its results.
\end{abstract}

\section{RESUMEN}

La anestesia espinal es una técnica ampliamente utilizada en la práctica clínica. Por lo general, el bloqueo nervioso está determinado por tres factores principales. El primero es la distribución del anestésico local en el líquido cefalorraquídeo (LCR), que a su vez se ve afectado por una gran variedad de factores, destacando entre estos el volumen de LCR. El segundo es la absorción, la cual es mayor en los sitios en donde la concentración del fármaco también lo es: para esto afecta la accesibilidad, el contenido lipídico y la irrigación vascular de

\section{Key words:}

Anesthesia, regional anesthesia, spinal anesthesia, pharmacokinetics

\section{Palabras clave:}

Anestesia, anestesia regional, anestesia espinal, farmacocinética

\section{ORCID}

0000-0002-0464-8643

Sin fuentes de financiamiento.

No se declaran conflictos de interés.

\section{Correspondencia:}

Fernando Altermatt Couratier

falterma@uc.cl 
cada zona. El último factor es la eliminación, mediada principalmente por la irrigación de los distintos compartimentos, y cuyo orden es distinto a la imagen especular del inicio de acción. Los factores mencionados son los principales determinantes de los tiempos de demora de los bloqueos, el orden en el que se logra su acción en las distintas fibras y el bloqueo diferencial. Este texto pretende describir los principales mecanismos de acción mediante los cuales actúa la anestesia espinal y los factores que pueden determinar diferencias en los resultados de esta.

\section{Introducción}

a inyección de anestésicos locales en el espacio subaracnoideo, en cantidad y concentración suficiente, produce interrupción de la transmisión neural desde la periferia hacia el sistema central y viceversa. Esto se traduce clínicamente en insensibilidad a estímulos sensitivos, parálisis motora y bloqueo de reflejos viscerales, permitiendo excelentes condiciones quirúrgicas.

El sitio de acción fundamental son las raicillas espinales que transitan por el espacio subaracnoideo entre la médula espinal y el saco dural[1]. En modelos animales, los estudios histológicos han medido niveles medulares de anestésicos incluso mayores que en nervios espinales, sugiriendo que éste también puede ser un sitio de acción[2]. Sin embargo, estudios fisiológicos con potenciales evocados, han demostrado que durante las anestesias espinales altas aún hay transmisión de información desde sitios medulares hacia el cerebro[3]. La experiencia clínica también apoya la no acción medular: en pacientes sometidos a anestesia espinal con punción a nivel toráxico T10, se logra un bloqueo segmentario sensitivo efectivo con un rango medio de T3 a L3 (máximo superior T2 y máximo inferior L5) a los 15 minutos con poco o ningún bloqueo distal en extremidades inferiores[4]. El bloqueo segmentario producido sugiere conservación de la función conductiva medular. Esto también podría explicarse por el desarrollo axorizomiélico, según el cual las fibras nerviosas que inervan estructuras proximales se ubican en una posición más periférica o superficial que las fibras distales. La penetración del anestésico local pudiera limitarse a concentraciones efectivas solo en la periferia y permitir la actividad normal más profunda.

Durante la instalación del bloqueo es posible observar diferencias en el tiempo de establecimiento de los bloqueos motor y sensitivo, más aún, diferencias en la instalación de distintas percepciones sensitivas. La clásica descripción es una secuencia de eventos clínicos en el siguiente orden: 1) vasodilatación cutánea y sensación de calor; 2) pérdida de la sensibilidad dolorosa y térmica; 3) pérdida de la sensibilidad de tacto y 4) bloqueo motor. Una vez establecido el nivel anestésico al pinchazo cutáneo (pin prick) es posible establecer bloqueo térmico y simpático a niveles más altos, lo que se conoce como bloqueo diferencial. Este comportamiento se explica por varias razones: en primer lugar, existen diferencias de sensibilidad entre fibras mielínicas y amielínicas. In vitro, en modelos de nervios en baño de anestésico, las fibras mielínicas son más sensibles que las fibras amielínicas[5]; en segundo lugar, existen diferencias de afinidad de los anestésicos locales por las fibras nerviosas. La etidocaína, por ejemplo, produce mayor bloqueo en fibras motoras que la bupivacaína[6]; en tercer lugar, los axones que presentan impulsos de alta frecuencia tienen mayor sensibilidad[7] y, por último, en el caso de las fibras mielínicas, el bloqueo depende de la capacidad de difusión axial para cubrir al menos tres nódulos de Ranvier consecutivos, ya que fibras más gruesas tienen mayor distancia entre los nodos[8], es decir, el largo del segmento internodal es más importante que el ancho de la fibra nerviosa, en términos de sensibilidad a los anestésicos locales.

En clínica se acostumbra a observar y medir el bloqueo de nervios somáticos motores y sensitivos. Sin embargo, junto con establecerse el bloqueo somático, también se produce interrupción de la inervación eferente visceral que regula total o parcialmente su funcionamiento.

\section{Mecanismo de acción}

Al inyectar soluciones anestésicas en el espacio subaracnoideo, las características del bloqueo anestésico será el resultado de tres factores mayores: 1) Distribución del anestésico local ( $\mathrm{AL}$ ) en el líquido cefalorraquídeo (LCR), que determina la extensión de la alteración en la función neuronal; 2) Absorción (up- 
take) por el tejido neural, que determina las funciones que se afectarán, y 3) eliminación, que determina la duración de la alteración neuronal[9].

El mecanismo de acción de la anestesia espinal es principalmente por el efecto de los anestésicos locales a nivel de los canales de $\mathrm{Na}^{+}$de las neuronas. Bajo condiciones ideales el bloqueo nervioso es un fenómeno todo-o-nada. Esto significa que los axones de un nervio que estén expuestos a una concentración mayor o igual a la concentración mínima para esa fibra $\left(C_{m}\right)$ asociado a un volumen suficiente para bloquear 3 o más nodos de Ranvier adyacentes, estará sólidamente anestesiado[10],[11]. Esto se expresa en la ecuación de "seguridad residual de conducción", propuesta por Fink[11]:

$$
S_{R}=1 / k F\left(C_{m} \bullet N_{A}\right)
$$

Donde $S_{R}$ es la seguridad residual de conducción (cuyo valor normal es 5 a 6 ), $k$ es una constante de potencia para cada anestésico, $\mathrm{F}$ es una constante dependiente de la difusión del anestésico en el LCR, su absorción neural y su eliminación sistémica. $C_{m}$ es la mínima concentración del anestésico local para bloquear un nodo de Ranvier y $\mathrm{N}_{\mathrm{A}}$ es el número de nodos consecutivos que baña el anestésico $\left(N_{A}\right.$ debe ser al menos 3). Si el valor de $S_{R}$ es menor a 1 , se produce bloqueo de conducción[11]. La ola de propagación del potencial de acción lo hace en distancias mucho mayores a su diámetro, del orden de 1.000 veces para fibras $C(1 \mathrm{~mm})$ y 3.000 a 5.000 veces para fibras A alfa (3-5 mm)[12]. Así, el potencial de acción que fluye por la fibra es 5-10 veces más potente que lo necesario para depolarizar el siguiente segmento, lo que se denomina margen de seguridad. Dado este margen, se requeriría bloquear $80 \%$ de los canales de $\mathrm{Na}^{+}$para evitar la propagación del estímulo. Se sabe que, si se utiliza una concentración que bloquee más de $84 \%$ de la conductancia al sodio, en tres nodos consecutivos, se previene toda propagación eléctri$\mathrm{ca}[11],[12]$.

Como complemento al modelo es el efecto anestésico de la temperatura fría. A diferencia de los anestésicos locales que interactúan y tapan los canales de $\mathrm{Na}^{+}$, las bajas temperaturas disminuyen la velocidad de conducción neuronal al enlentecer la apertura de dichos canales[13].

Para acceder a las fibras nerviosas, el anestésico una vez depositado en el espacio subaracnoideo, debe distribuirse (ver abajo) para entrar en contacto con las neuronas. La absorción del AL por el tejido neural (uptake) dependerá de cuatro factores operativos simultáneos: accesibilidad, concentración del AL, contenido lipídico y flujo sanguíneo tisular[2]. En consecuencia, el anestésico tendrá varios sitios probables de acceso al tejido neural, que nos son excluyentes, pero que tienen un orden preferencial.

1. Médula espinal: la absorción (uptake) de los anestésicos intratecales ocurre preferentemente en los cordones posteriores y laterales de la médula espinal.

a. Difusión: el AL difundirá a través de la piamadre y lo hará unos pocos milímetros en la médula espinal en forma directa. Este es un proceso lento.

b. Espacio de Virchow-Robin: el anestésico se introducirá al espesor de la médula espinal por los espacios de Virchow-Robin, que son las hendiduras que acompañan a los vasos sanguíneos que ingresan a la médula espinal. Estos están recubiertos por piamadre igualmente (Figura 1).

2. Raicillas nerviosas: el anestésico hará efecto en las raicillas nerviosas que transitan por el saco dural, desde que emergen de la médula espinal hasta el agujero de conjunción cuando se unen las raicillas dorsales y ventrales. Las raicillas dorsales captan mayor cantidad que las ventrales. Es el lugar don-

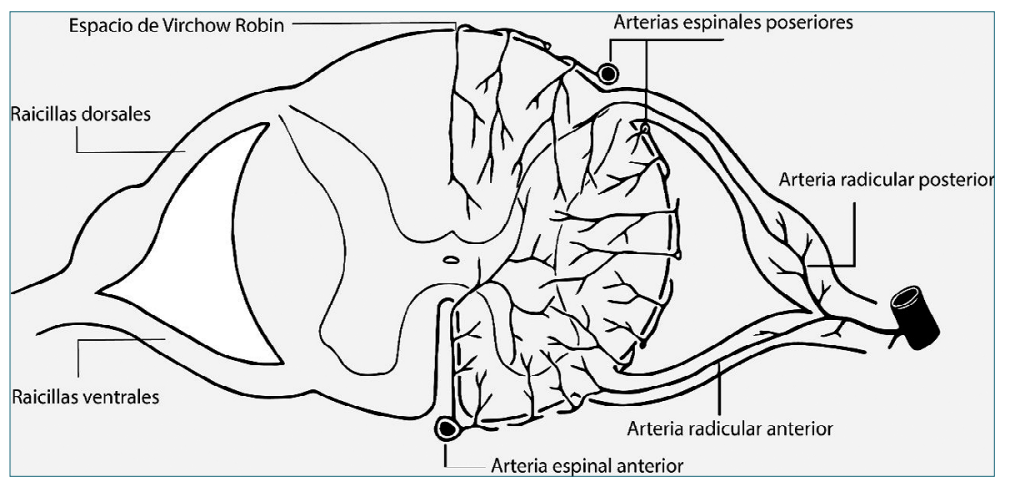

Figura 1. 
de se ejerce principalmente el efecto anestésico de la técnica espinal.

Las características de los nervios le confieren mayor o menor resistencia al efecto anestésico local. La mielina le confiere una mayor protección y resistencia, mientras que la distancia entre nodos de Ranvier, genera una resistencia agregada. Tradicionalmente, se dice que el grosor es un factor determinante y a mayor grosor, mayor resistencia. Sin embargo, esto no es así, tal como lo demostró Fink y cols[13],[14]. Si bien las fibras A delta y C (que llevan la información de dolor, temperatura y nocicepción) difieren en diámetro, mielinización y velocidad de conducción, su respuesta a los anestésicos locales es similar y su $C_{m}$ es prácticamente idéntica[10]. Sin embargo, a medida que aumenta el tamaño de las fibras nerviosas mielínicas, la probabilidad de bañar al menos 3 nodos de Ranvier se hace cada vez menor, por lo que el factor $\mathrm{N}_{\mathrm{A}}$ se altera y permite explicar el fenómeno de bloqueo diferencial que frecuentemente se comprueba en clínica[10].

Durante la mielinización que ocurre en el desarrollo, la distancia internodal es uniformemente corta (150-300 um), donde cada internodo representa el territorio de una célula de Schwann individual. A medida que el individuo crece, el número de nodos permanece constante, pero los internodos crecen en longitud, manteniéndose una proporción entre la distancia internodal y el crecimiento en largo donde ese nervio reside[10].

Como ejemplo, la distancia internodal de las fibras A delta va de 0,3 a 0,7 mm, mientras que la de las $A$ alfa es de 0,8 a $1,4 \mathrm{~mm}$. Por cada $1 \mu \mathrm{m}$ de grosor, aumenta la distancia internodal en 0,7 mm[10]. Las fibras A alfa tendrán una distancia crítica de bloqueo de al menos $5 \mathrm{~mm}$ y la $C_{m}$ es esencialmente idéntica para ambas fibras. En consecuencia, un baño de $2 \mathrm{~mm}$ de $\mathrm{AL}$ bloqueará las fibras $A$ delta mientras que las $A$ alfa no se verán afectadas, produciéndose un bloqueo diferencial (analgesia sin bloqueo motor). Así, más que el grosor, importa el largo de la distancia internodal en las fibras mielínicas, aumentando su velocidad de conducción en 5-6 m/seg por cada 1 um de grosor[10]. Es decir, el "principio del grosor" (a mayor grosor de la fibra, más difícil de bloquear), ha sido reemplazado por el "principio del largo" (a menor número de nodos, más difícil de bloquear)[10].

Para el caso de un bloqueo epidural, el número de nodos de Ranvier bloqueados a nivel lumbar será numeroso y, por lo tanto, no será un factor determinante del bloqueo. Sin embargo, a niveles más altos, pudiera ser un factor determinante al no alcanzarse necesariamente a bloquear 3 nodos con una $C_{m}$ mínima, generándose así la posibilidad de bloqueo diferencial.

\section{Distribución}

Se han identificado al menos 25 factores que potencialmente pueden afectar la distribución del AL en el espacio subaracnoideo (Tabla 3)[15]. Algunos de los factores que han sido razonablemente descartados como clínicamente significativos en la distribución del anestésico local son: sexo, barbotage, difusión del $A L$ en el LCR, composición del LCR, presión del LCR, concentración del anestésico local y el uso de vasoconstrictores. Aquellos factores que claramente tienen un rol en la distribución son: edad, altura, configuración anatómica de la columna, sitio de inyección, dirección de la aguja, volumen de LCR, densidad del LCR, baricidad del AL, posición del paciente, dosis y volumen del anestésico inyectado (se ha visto que, si se inyecta un volumen mayor a $10 \%$ de volumen total de LCR, este inyectado se detectará en el cerebro)[16]. Finalmente, aún son controversiales (aunque perfectamente posibles) el rol del peso del paciente[17], dirección del bisel, velocidad de inyección[16], pulsatilidad del LCR (amplitud y frecuencia, con movimiento longitudinal del LCR entre 4-9 mm por cada ciclo)[12],[18], microanatomía intratecal, circulación del LCR, velocidad de inyección[16] y uso de la técnica de extensión con volumen epidural (EVE)[19].

Especial consideración tiene el volumen de LCR. Este da cuenta de $80 \%$ de la variabilidad observada en el pico de ascenso y la regresión de un bloqueo espinal. De hecho, estudios han demostrado que el parámetro que mejor describe el resultado de un bloqueo espinal en cuanto a su altura es el volumen del LCR. Medido por resonancia nuclear magnética, el volumen del LCR puede ser muy variable[20],[21]. No existe una buena correlación entre este parámetro con peso o talla del individuo. Desafortunadamente, la medición clínica del volumen de LCR no es posible.

\section{Absorción (uptake)}

La distribución del AL determina la absorción que ocurrirá en el tejido neural en el canal espinal[2]. Esta será mayor donde exista mayor concentración del anestésico. La magnitud del bloqueo será mayor donde exista más absorción y decrecerá en función de la distancia del sitio de inyección. Esto implica que la anestesia espinal inevitablemente tendrá una graduación en la denervación, con la posibilidad de un 
bloqueo diferencial.

La absorción por los diferentes tejidos neurales dependerá de 3 factores: 1) accesibilidad; 2) contenido lipídico y 3) flujo sanguíneo tisular. A nivel espinal, si bien las raicillas nerviosas son las más expuestas al anestésico local disuelto en el LCR (tejido con la ma- yor accesibilidad), no son las estructuras con la mayor concentración del anestésico (Tabla 1), explicado por el importante rol de los otros dos factores.

La médula espinal tiene amplia accesibilidad al AL disuelto en el LCR. La accesibilidad es función de los espacios de Virchow-Robin. Estos acompañan a los

\begin{tabular}{|c|c|c|c|c|c|}
\hline Fibra & Mielinizada & $\begin{array}{l}\text { Diámetro } \\
\text { (um) }\end{array}$ & $\begin{array}{l}\text { Velocidad } \\
\text { (m/seg) }\end{array}$ & $\begin{array}{c}\text { Distancia } \\
\text { internodal }(\mathrm{mm})\end{array}$ & Función \\
\hline A alfa & + & $15-20$ & $80-120$ & $0,8-1,4$ & Motor, propriocepción \\
\hline A beta & + & $8-15$ & 90 & & Motor, tacto y $\mathrm{T}^{\circ}$ \\
\hline A gama & + & $4-8$ & 50 & & Tono muscular \\
\hline A delta & + & $3-4$ & $10-15$ & $0,3-0,7$ & Dolor y $\mathrm{T}^{\circ}$ \\
\hline B & + & 4 & $10-15$ & & Preganglionares autonómicas \\
\hline C & - & $1-2$ & $1-2$ & - & Dolor y $\mathrm{T}^{\circ}$ \\
\hline
\end{tabular}

La velocidad de conducción de las fibras tipo $\mathrm{A}$ es $\sim 6 \mathrm{x}$ el diámetro. La velocidad de conducción de las fibras tipo A delta es similar a la de las fibras $C$ amielínicas. La distancia internodal aumenta con el grosor de la fibra por un factor de $\sim 100 x$.

Tabla 2. Concentración de lidocaína en la médula espinal lumbar de 6 animales, 30 minutos después de la inyección de 25 mg de lidocaína en $1 \mathrm{ml}$ (15) en el espacio subaracnoídeo

\section{Sección}

Cordón lateral

Cordón posterior

Raicillas dorsales

Cordón anterior

Sustancia gris

Raicillas ventrales

Ganglio de la raíz dorsal
Concentración del AL (ug/mg de tejido \pm error estándar)

$$
\begin{gathered}
1,38 \pm 0,12 \\
1,36 \pm 0,18 \\
0,87 \pm 0,20 \\
0,73 \pm 0,24 \\
0,53 \pm 0,09 \\
0,32 \pm 0,10 \\
0,16
\end{gathered}
$$

\begin{tabular}{llll}
\multicolumn{2}{l}{ Tabla 3. Factores que influyen en la distribución de soluciones de anestésico local en el líquido cefalorraquídeo } \\
(LCR)
\end{tabular}


vasos sanguíneos que ingresan a la médula (Figura 1). Como la irrigación medular es derivada principalmente por la arteria espinal anterior, habrá mayor disponibilidad de estos espacios por anterior. Sin embargo, la mayor concentración de los anestésicos locales se encuentra en los cordones posteriores y laterales, principalmente por el rol del contenido lipídico. Los anestésicos locales tradicionales son altamente solubles en lípidos. La mielina contiene lípidos y los cordones mencionados están más mielinizados que los anteriores (a excepción del tracto corticoespinal anterior)[2].

La concentración tisular de los anestésicos locales también estará gobernada por el flujo sanguíneo tisular, ya que este determina la tasa a la cual el anestésico es removido del tejido. A mayor flujo sanguíneo por unidad de tejido, más rápido es removido el anestésico. Así, el mayor flujo sanguíneo de los cordones anteriores, sumado a una menor mielinización comparado con los posteriores, explicaría la mayor concentración alcanzada en estos.

La traducción clínica de lo anterior es que luego de una inyección de AL en el espacio subaracnoideo, la secuencia de bloqueo será en el siguiente orden: temperatura y dolor (fibras A delta), luego las fibras $B$, antes que las $C$, posiblemente, porque estas últimas están rodeadas de células de Schwann en los llamados Manojos de Remak que impiden el acceso de AALL fácilmente y los intervalos internodales de las fibras B son cortos[22]. Luego se bloquearán las fibras $C$, seguidas de una denervación simpática preganglionar (fibras A gama), fibras sensitivas somáticas (fibras A beta) y luego las fibras motoras somáticas (fibras $A$ alfa). Las últimas fibras en bloquearse serán aquellas en tractos dentro de la médula espinal, como se mencionó previamente.

\section{Eliminación}

Los anestésicos locales son removidos del espacio subaracnoideo por absorción vascular intratecal, vascular medular y por difusión a través de la duramadre-aracnoides hacia el espacio epidural, donde habrá absorción vascular epidural. La tasa de absorción vascular de los tres compartimientos está inversamente relacionada a la solubilidad lipídica. Es interesante mencionar que la eliminación no depende de la metabolización de los anestésicos locales en el LCR[2].

La secuencia en la que se recupera un bloqueo neural no es una imagen especular del inicio de acción. El inicio de acción depende de la accesibilidad y del contenido lipídico del tejido a ser bloqueado, mientras que la recuperación depende principalmente de la perfusión vascular[2].

\section{Referencias}

1. Greene NM, Brull SJ. Physiology of Spinal Anesthesia. 4th ed. Baltimore: Williams \& Wilkins; 1993. 387 pp.

2. Greene NM. Uptake and elimination of local anesthetics during spinal anesthesia. Anesth Analg. 1983 Nov;62(11):1013-24. https:// doi.org/10.1213/00000539198311000-00011 PMID:6354003

3. Lang E, Krainick JU, Gerbershagen HU. Spinal cord transmission of impulses during high spinal anesthesia as measured by cortical evoked potentials. Anesth Analg. 1989 Jul;69(1):15-20. https:// doi.org/10.1213/00000539-
198907000-00004

PMID:2742163

4. van Zundert AA, Stultiens G, Jakimowicz JJ, Peek D, van der Ham WG, Korsten HH, et al. Laparoscopic cholecystectomy under segmental thoracic spinal anaesthesia: a feasibility study. $\mathrm{Br}$ J Anaesth. 2007 May;98(5):6826. https://doi.org/10.1093/bja/ aem058 PMID:17371777

5. Gissen AJ, Covino BG, Gregus J. Differential sensitivities of mammalian nerve fibers to local anesthetic agents. Anesthesiology. 1980 Dec;53(6):467-74. https:// doi.org/10.1097/00000542198012000-00006 PMID:7457962

6. Gissen AJ, Covino BG, Gregus J. Differential sensitivity of fast and slow fibers in mammalian nerve. III. Effect of etidocaine and bupivacaine on fast/ slow fibers. Anesth Analg. 1982 Jul;61(7):570-5. https:// doi.org/10.1213/00000539198207000-00004 PMID:6283948

7. Courtney KR, Kendig JJ, Cohen EN. Frequency-dependent conduction block: the role of nerve impulse pattern in local anesthetic potency. Anesthesiology. 1978 Feb;48(2):111-7. https:// doi.org/10.1097/00000542197802000-00006 PMID:306789

8. Raymond SA, Steffensen SC, Gugino LD, Strichartz GR. The role of length of nerve exposed to local anesthetics in impulse blocking action. Anesth Analg. 
1989 May;68(5):563-70. https:// doi.org/10.1213/00000539198905000-00004

PMID:2785778

9. Stienstra R, Greene NM. Factors affecting the subarachnoid spread of local anesthetic solutions. Reg Anesth. 1991 JanFeb;16(1):1-6. PMID:2007097

10. De Jong RH. Differential Nerve Block. In: Anesthetics L, editor. Mosby. St Louis: First; 1994. pp. 81-97.

11. Fink BR. Mechanisms of differential axial blockade in epidural and subarachnoid anesthesia. Anesthesiology. 1989 May;70(5):851-8. https:// doi.org/10.1097/00000542198905000-00023 PMID:2719320

12. Hogan Q. Anatomy of the Neuraxis. In: Cousins M, Carr D, Horlocker T, Bridenbaugh $\mathrm{P}$, editors. Neural Blockade In Clinical Anesthesia and Pain Medicine. 4th ed. Philadelphia: Lippincott Williams \& Wilkins; 2009. pp. 181-212.

13. Fink BR, Cairns AM. Differential slowing and block of conduction by lidocaine in individual afferent myelinated and unmyelinated axons. Anesthesiology. 1984 Feb;60(2):111-20. https:// doi.org/10.1097/00000542198402000-00006 PMID:6696232

14. Fink BR, Cairns AM. Lack of size-related differential sensitivity to equilibrium conduction block among mammalian myelinated axons exposed to lidocaine. Anesth Analg. 1987 Oct;66(10):948-53. https:// doi.org/10.1213/00000539198710000-00004 PMID:3631589

15. Greene NM. Distribution of local anesthetic solutions within the subarachnoid space. Anesth Analg. 1985 Jul;64(7):715-30. https:// doi.org/10.1213/00000539198507000-00012 PMID:3893222

16. Tangen KM, Leval $R$, Mehta Al, Linninger AA. Computational and in vitro experimental investigation of intrathecal drug distribution: parametric study of the effect of injection volume, cerebrospinal fluid pulsatility, and drug uptake. Anesth Analg. 2017 May;124(5):1686-96. https://doi.org/10.1213/ ANE.0000000000002011 PMID:28431428

17. Ngaka TC, Coetzee JF, Dyer $\mathrm{RA}$. The influence of body mass index on sensorimotor block and vasopressor requirement during spinal anesthesia for elective cesarean delivery. Anesth Analg. 2016 Dec;123(6):1527-34. https://doi.org/10.1213/ ANE.0000000000001568 PMID:27870737

18. Hsu Y, Hettiarachchi HD, Zhu DC, Linninger AA. The frequency and magnitude of cerebrospinal fluid pulsations influence intrathecal drug distribution: key factors for interpatient variability. Anesth Analg. 2012 Aug;115(2):38694. https://doi.org/10.1213/ ANE.0b013e3182536211 PMID:22523420

19. Takiguchi T, Okano T, Egawa H, Okubo Y, Saito K, Kitajima $T$. The effect of epidural saline injection on analgesic level during combined spinal and epidural anesthesia assessed clinically and myelographically. Anesth Analg. 1997 Nov;85(5):1097-100. https:// doi.org/10.1213/00000539199711000-00024 PMID:9356106

20. Hogan $Q H$, Prost $R$, Kulier $A$, Taylor M, Liu S, Mark L. Lou, Liu S, Mark L. Magnetic resonance imaging of cerebrospinal fluid volume and the influence of body habitus and abdominal pressure. Anesthesiology. 1996;84(6):1341-9. https:// doi.org/10.1097/00000542199606000-00010.

21. Sullivan JT, Grouper S, Walker MT, Parrish TB, McCarthy RJ, Wong CA. Lumbosacral cerebrospinal fluid volume in humans using threedimensional magnetic resonance imaging. Anesth Analg. 2006 Nov; 103(5):1306-10. https://doi.org/10.1213/01. ane.0000240886.55044.47 PMID:17056974

22. De Jong RH. Threshold-blocking Phenomena. In: Anesthetics L, editor. Mosby. St Louis: First; 1994. pp. 64-80. 